\title{
Linear and Directional Domains with Cauchy Probability Distributions
}

\author{
T.D. Downs ${ }^{*}$ and K.J. Downs
}

Research Statistics, Inc., P.O. Box 840597, Houston, Texas 77284-0597, U.S.A

\begin{abstract}
The usual domains for Cauchy distributions have been straight lines and unit circles. These domains are closed under arbitrary changes in location and scale, whether done sequentially or simultaneously. Such closure properties have been extended to spherical Cauchy distributions. Higher dimensional Cauchy-based domains are created herein for unit hyperspheres and sets of straight lines of arbitrary dimension, and their Cauchy-like properties are determined and described. Cauchy distributions on these extended domains are shown to be closed under arbitrary transformations of location and scale, done singly or sequentially, but not generally closed when location and scale changes are done simultaneously. Stereographic projections are used to map the curved, finite surface of any hypersphere to a linear, infinite space of the same dimension as that of the hyperspherical surface. These mappings are one-one and onto, with no loss of information. These results show promise for uniting linear and directional mixtures of observations into a common domain-linear or directional.
\end{abstract}

Keywords: Euclidean space, Fisher-von Mises distribution, Generalized stereographic projection, Hyperspheres, Image analysis, Möbius mapping, Pattern recognition.

\section{INTRODUCTION}

Let $x_{1} \ldots x_{\mathrm{n}}$ be a rectangular coordinate system for an $e x$ tended Euclidean space $\mathrm{E}_{\mathrm{n}}$ (n-space, for short). The "extended" feature means that the n-space has appended to it the single improper point $\infty$. That is, $-\infty<x_{\mathrm{j}} \leq \infty$ for $\mathrm{j}=1-n$. This results, as will be seen, in the ability to transform directional data back and forth between curved and linear spaces with the same dimensionality with no loss of information, via generalized stereographic projection.

Row vectors are used for brevity: $0=(0 \ldots 0)$ is the $n$ vector origin in $\mathrm{E}_{\mathrm{n}}$ and $x=\left(x_{1} \ldots x_{\mathrm{n}}\right)$ is a typical point of $\mathrm{E}_{\mathrm{n}}$. The equation $(x-c)(x-c)^{\mathrm{T}}=r^{2}$ defines a hypersphere $\mathrm{E}_{\mathrm{n}}$ with center $c=\left(c_{1} \ldots c_{\mathrm{n}}\right)$ and radius $r$.

Our focus is on unit hyperspheres, where $c=0$ and $r=|x|$ $=1$. Our aim is to create and describe families of Cauchybased distributions on linear and spherical surfaces of arbitrary dimension.

Hyperspheres are classified by the dimensionality, $m$, of their surfaces. A circle $C$ has $m=1$ but, as a courtesy, it is called a 1-sphere or $S_{1}$. An ordinary sphere with 2dimensional surface area is a 2-sphere or $S_{2}$ with $m=2$, and a hypersphere with generic $m$-dimensional surface content is an $m$-sphere $S$ or $S_{\mathrm{m}}$.

An $m$-sphere needs at least $m+1$ dimensions to properly display itself and to provide the additional dimension needed for rectangular coordinates (Fig. 1). So, we put $n=m+1$, and let $n$ vary as $m$ varies at will. Dimension $n$ is not to be confused with the non-italicized north pole $n$ of a sphere or hypersphere.

*Address correspondence to this author at the Research Statistics, Inc., P.O. Box 840597, Houston, Texas, U.S.A; Tel: 77284-0597;

E-mail: proftddowns@msn.com

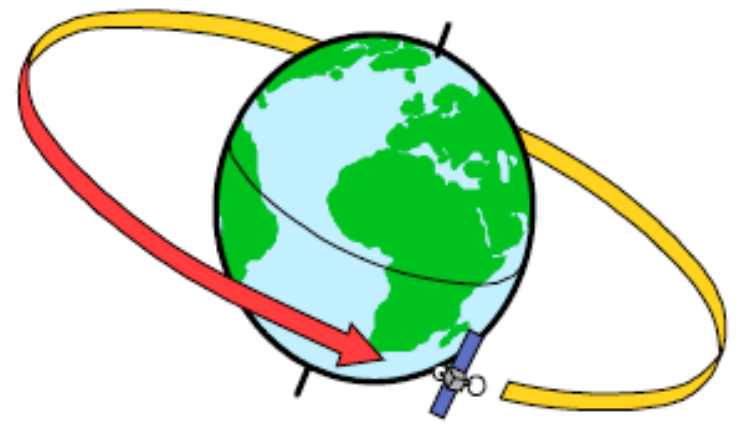

Fig. (1). Satellite orbiting a 2-sphere $S_{2}$ in $\mathrm{E}_{3}$. A sensor at the center of $S_{2}$ can record the spatial direction to the satellite or to any other detectable object in 3-space by the rectangular or spherical coordinates of the point where the "line of sight" from the center of the sphere to the center of the satellite intersects $S_{2}$. Directions in $n$-space may be recorded as the coordinates of points on an $m$-sphere $S_{\mathrm{m}}$ in $n$-space $(n=m+1)$. If the sphere were a 1 -sphere $S_{1}$ in $\mathrm{E}_{3}$ instead of a 2 -sphere the universe of detectable objects would be limited to those in the plane of $S_{1}$.

The default notation for a circle is $C$ or $S_{1}$, that for a $d$ sphere is $S_{\mathrm{d}}$ when $d$ is not $m$, and that for an $m$-sphere is $S$ or $S_{\mathrm{m}}$.

Geographic terminology is used: to refer to the various hemispheres; to the equator and its equatorial $m$-space $\mathrm{E}_{\mathrm{m}}$; to the north and south poles $n=(10 \ldots 0)$ and $s=-n$; to the vertical polar axis through the poles and coincident with the $x_{1}$ axis; to colatitude $\theta$ (the angular deviation of a point $x$ on an $m$-sphere from a special fixed point on the $m$-sphere); to the polar circles passing through the poles $\mathrm{n}$ and $\mathrm{s}$, and their horizontal centerlines L passing through the origin.

The default notation for a single centerline is $\mathrm{L}$ or $\mathrm{L}_{1}$, that for a $d$-space of centerlines is $L_{\mathrm{d}}$ when $d$ is not $m$, while that for an $m$-space of centerlines is $L$ or $L_{\mathrm{m}}$. This $m$-space is a subspace of $E_{n}$, and is therefore itself an extended $E_{m}$. 
An $m$-sphere $S$ generated by a sequence of rotations is created in $\S 2$. The sequence begins by rotating a circle in $2-$ space into a sphere in 3-space, and ends with the generation of an $m$-sphere in $n$-space by rotation of an $(m-1)$-sphere. Section 3 defines and describes certain terms and probability distributions, and gives some details for transforming distributions on $m$-sphere surfaces to and fro between rectangular $x=\left(x_{1} \ldots x_{\mathrm{n}}\right)$ coordinates and angular or spherical coordinates $a=\left(r a_{1} \ldots a_{\mathrm{m}}\right)$.

Section 4 lays the groundwork for methodology to change back and forth between linear and directional domains. Section 5 expands the methodology of $\S 4$ to perform sequential or simultaneous transformations in location and scale for Cauchy-based probability distributions on infinite linear and finite curved objects. Section 6 briefly discusses some potential applications of the methods proposed herein.

\section{CREATING M-SPHERES $S_{M}$ IN $E_{N}$}

Start with the unit circle $S_{1}$ in the $x_{1} x_{2} 2$-space of $\mathrm{E}_{\mathrm{n}}$, with the vertical $x_{1}$-axis as the polar axis through $\pm \mathrm{n}$, and the horizontal $\mathrm{x}_{2}$-axis as the centerline $\mathrm{L}_{1}$ for $S_{1}$.

Imagine being in $x_{1} x_{2} x_{3} 3$-space above $S_{1}$, looking down on the north pole n. Picture $S_{1}$ and its center line $\mathrm{L}_{1}$ rotating CCW (counter-clockwise) through an amount $\pi$ about the polar axis, and out of the $x_{1} x_{2} 2$-space containing $S_{1}$ into the 3 -space of $x_{1} x_{2} x_{3}$. This rotation creates a unit 2-sphere $S_{2}$ as a 2 -space surface of revolution that is a continuum of polar circles, and a continuum $L_{2}$ of their centerlines.

Continue this process by rotating $S_{2}$ and its continuum of centerlines CCW through the same amount $\pi$ about the same polar axis but now out of the 3 -space containing $S_{2}$ and into the 4-space of $x_{1} x_{2} x_{3} x_{4}$, thereby generating a 3 -sphere $S_{3}$ and a 2 -fold continuum $L_{3}$ of centerlines.

Continue this pattern, creating $S_{4}$, $S_{5}$ etc., and their associated continuums of centerlines, and finishing with the creation of $S_{\mathrm{m}}$ and an $(m-1)$-fold continuum $L=L_{\mathrm{m}}$ of centerlines.

The poles, $\pm \mathrm{n}$, of any $m$-sphere $S$ are considered as fixed and immutable, with $\mathrm{n}$ at the top of the polar axis, $\mathrm{s}$ at the bottom, and the polar axis orthogonal to the equatorial $m$-space $\mathrm{E}_{\mathrm{q}}$ spanned by $x_{2} \ldots x_{\mathrm{n}}$. The $m$-space $L_{\mathrm{m}}$ spanned by all the centerlines of $S$ is the same space as the equatorial space $\mathrm{E}_{\mathrm{q}}$.

\section{CAUCHY M-SPHERE PARAMETERS}

\subsection{Mean Direction $\mu$ and Rescaler $\delta$}

All Cauchy-based directional distributions dealt with herein have two parameters in addition to their dimension. These are the unit $n$-vector location parameter, $\mu$, and the non-negative scale parameter $\delta$, called the rescaler. The points $x$ and $\mu$ on an $m$-sphere $S$ have the vector forms:

$x=\left(x_{1} \ldots x_{\mathrm{n}}\right), \mu=\left(\mu_{1} \ldots \mu_{\mathrm{n}}\right)$, with $|\mathrm{x}|=|\mu|=1$.

The mean $\mu$ of a Cauchy $m$-sphere is the unit $n$-vector from the origin to the center of gravity, $\mathrm{E}(x)$, of the probability distribution for $x \in S_{\mathrm{m}}$. This center of gravity is the origin, 0 , when the mean direction does not exist. We use the improper value 0 for $\mu$ to represent this, and write

$\mathrm{E}(x)=\rho \mu$, where $0 \leq \rho \leq 1$, and $\mu \in S \Leftrightarrow \mu \neq 0$.
The length $\rho$ of $\mathrm{E}(x)$ is called the precision. It is shown later that a Cauchy sphere $S$ is uniformly distributed if and only if the rescaler $\delta=1$, in which case the precision $\rho=0$, and $\mu=0$. By default $\delta \neq 1$ and $\rho \neq 0$. However, if $\delta=0$ or $\infty$ then $\rho=1$, and $x=\mu$ with probability one.

For circles, $\delta$ and $\rho$ are related $[1 ; \S 3.3]$ ) by

$\rho(\delta)=|1-\delta| /(1+\delta)=\rho(1 / \delta)$ for any $\delta>0$.

This formula changes with $m$, becoming more complicated for larger $m$, e.g.: when $m=2, \rho=\operatorname{coth} \kappa-1 / \kappa$ where $\delta$ $=\exp (\kappa)[1 ; \S 4.4]$.

\subsection{Quick Definition of $S_{\mathrm{m}}$ Distribution}

The $m$-sphere $S$ is said to be Cauchy-based when the variable part of its probability density function (also called the profile graph function, or $\mathrm{pgf}$ for short) has the form: $\operatorname{pgf}(\theta)$ $\propto(A-B \cos \theta)^{-1}$, in which case we write:

$S$, or $x$, or $\theta \sim \mathrm{C}_{\mathrm{m}}(\mu . \delta)$

where $A$ and $B$ are constants and functions of $\delta ; \cos \theta=$ $x \mu^{\mathrm{T}}$ where $x$ is a point on $S, \mu$ is the mean direction for $x$, and $\theta$ is the colatitude for $x$. In such case we say $S$, or $x$, or $\theta$ is Cauchy $\mu, \delta$. When $S$ has a uniform distribution we say $S$ is Cauchy 0,1 , and write: $S$, or $x$, or $\theta \sim \mathrm{C}_{\mathrm{m}}(0,1)$.

\subsection{Transforming Coordinate Systems}

The equations given in this section for transforming spherical to rectangular coordinates for points on $m$-spheres were adapted from [2]. They are used below to derive the Jacobian matrix $\mathrm{J}$ for the transformation.

Assume for the moment that the radius $r$ of $S$ is arbitrary. For brevity put $s_{\mathrm{d}}=\sin a_{\mathrm{d}}$ and $c_{\mathrm{d}}=\cos a_{\mathrm{d}}$, for $d=1-m$. The elements of $x$ are given, in terms of those for the spherical coordinates in $a=\left(r a_{1} \ldots a_{\mathrm{m}}\right)$, by:

$x_{1}=r \times c_{1} ; x_{\mathrm{d}}=r \times s_{1} s_{2} \ldots s_{\mathrm{d}-1} c_{\mathrm{d}}$, for $d=2-m ; \quad x_{\mathrm{n}}=r \times$ $s_{1} s_{2} \ldots s_{\mathrm{m}-1} s_{\mathrm{m}}$.

Equations for expressing the spherical coordinates of $a$ in terms of the rectangular coordinates of $x$ are given in [2]. The angle $a_{\mathrm{m}}$, given by $a_{\mathrm{m}}=2$ a cot $\mathrm{q}$, where $\mathrm{q}=\left\{\left(\mathrm{x}_{\mathrm{m}}{ }^{2}+\mathrm{x}_{\mathrm{n}}{ }^{2}\right)^{1 / 2}\right.$ $\left.+\mathrm{x}_{\mathrm{m}}\right) / \mathrm{x}_{\mathrm{n}}$, plays the role of a longitude for $S$. For further details see [2].

\subsection{Jacobian of the Transformation}

The radius $r$ of $S$ is allowed to vary. $S$ has a uniform distribution on its surface and rectangular coordinates $x$. The joint probability density function ( $p d f$, for short) for $x$, when $S \sim \mathrm{C}_{\mathrm{m}}(0,1)$, is: $p d f(x)=$ a norming constant. The joint $p d f$ $(\mathrm{x})$ is to be transformed to the more tractable joint $p d f(a)$ of the angular coordinates in $a$ for $S$. The $n \times n$ Jacobian matrix J of partial derivatives is defined by its row-column element $k, d$, or its column $d$, from

$\mathrm{J}=\left(\partial x_{\mathrm{k}} / \partial a_{\mathrm{d}}\right)$, or $\mathrm{J}=\left(\partial x / \partial a_{0} \partial x / \partial a_{1} \ldots \partial x / \partial a_{\mathrm{m}}\right)$

The change of variables theorem [3] provides that the joint $p d f(a)$ is related to the joint $p d f(x)$ for all $x$, except the origin 0 , by:

$p d f(a)=p d f\{x(a)\}|\operatorname{det} \mathrm{J}$, 
where det $\mathrm{J}$ is found to be

det $\mathrm{J}=\mathrm{a}_{0}{ }^{\mathrm{m}} \sin ^{\mathrm{m}-1} a_{1} \sin ^{\mathrm{m}-2} a_{2} \ldots \sin ^{1} a_{\mathrm{m}-1}$

Now fix $a_{0}=r=1$. Since the distribution on $S$ is uniform, $p d f\{x(a)\}$ is also just a norming constant, say $N$, and (3) can be expressed as

$p d f(a)=\Pi_{\mathrm{d}} p d f\left(a_{\mathrm{d}}\right)=\Pi_{\mathrm{d}}\left(N_{\mathrm{d}} \sin ^{\mathrm{m}-\mathrm{d}} a_{\mathrm{d}}\right), d=1-m$

with $N_{\mathrm{d}}$ a norming constant for $a_{\mathrm{d}}$ and $N=N_{1} \times \ldots \times N_{\mathrm{m}}$. The $m^{\text {th }}$ sine term of the second product in (5) has disappeared since $\sin ^{\mathrm{m}-\mathrm{m}} a_{\mathrm{m}} \equiv 1$. Conclude from (5) that the $m$ angles $a_{\mathrm{j}}$ are jointly independent and uniformly distributed over their respective surfaces, which fixes the norming constants $N_{\mathrm{d}}$ and $N$.

\subsection{Cauchy $m$-Sphere Distributions}

An $m$-sphere $S$ with a Cauchy distribution on its surface will be called a Cauchy $m$-sphere. It is defined by its probability density element (pde, for short), in (8) below. Let $S$ be a Cauchy $m$-sphere with mean $\mu$ and rescaler $\delta$, and put, as in §3.2:

$\cos \theta=x \mu^{\mathrm{T}}, 0 \leq \theta \leq \pi$; and $x, \mu \in S$, where $\theta$ is the (6) colatitude.

We say $S$ or $\theta$ or $x$ is Cauchy $\mu, \delta$, and write:

$S \sim \mathrm{C}_{\mathrm{m}}(\mu, \delta)$, or $\theta \sim \mathrm{C}_{\mathrm{m}}(\mu, \delta)$, or $x \sim \mathrm{C}_{\mathrm{m}}(\mu, \delta)$

when the $p d e$ for the colatitude $\theta$ has the form:

$p d e(\theta ; \mu, \delta)=K_{\mathrm{m}}(A-B \cos \theta)^{-1} \sin ^{\mathrm{m}-1} \theta \mathrm{d} \theta$

where $A$ and $B$ are functions of $\delta(9)$, and $K_{\mathrm{m}}$ is a norming constant which is a function of $m$ and $\delta$ :

$A=\left(\delta^{2}+1\right) /(2 \delta)>B=\left(\delta^{2}-1\right) /(2 \delta) \geq 0$

Equations (6)-(8) show that the $\mathrm{C}_{\mathrm{m}}(\mu, \delta)$ distribution is completely described by that for the scalar colatitude $\theta$. Formulae for integrating (8) to get $K_{\mathrm{m}}$ can be found in [4].

The probability density function ( $p d f$, for short) for $\theta$ is just $p d e(\theta)$ without the $\mathrm{d} \theta$ :

$p d f(\theta ; \mu, \delta)=\mathrm{K}_{\mathrm{m}}(A-B \cos \theta)^{-1} \sin ^{\mathrm{m}-1} \theta$,

and a profile graph function ( $p g f$ ) for $\theta$ is just $p d f(\theta)$ without the $\sin ^{\mathrm{m}-1} \theta$ :

$\operatorname{pgf}(\theta ; \mu, \delta) \propto(A-B \cos \theta)^{-1}$

By (9) and (11) the $\mathrm{C}_{\mathrm{m}}(\mu, \delta)$ distribution degenerates to $\mathrm{C}_{\mathrm{m}}(0,1)$ if and only if the rescaler $\delta$ is unity.

The $p g f$ has the same form for all $d$-spheres, regardless of their surface dimensions or mean directions. A similar constancy of the $p g f$ across different dimensions holds for the totality of Fisher-von Mises distributions [5], wherein $p g f$ $(\theta)=\exp (\kappa \cos \theta)$

Any function proportional to (11) also serves as a $p g f$ for $\theta$. Knowing $m$ and the form of one of the $p d e, p d f$ or $p g f$ functions of (8), (10) or (11), respectively, is equivalent to knowing all three forms.

Contours of equal pgf values are the parallel (m-1)spheres of latitude centered about the mean axis. These con- tour values decrease monotonically from a maximum at the mode $\mu$, where $\theta=0$, to a midpoint when $\theta=\pi / 2$, to a minimum at $-\mu$, where $\theta=\pi$. On the other hand the $p d f$ values when $m \geq 2$ are zero at $\theta=0$, then climb rapidly, then decrease slowly to 0 again at $\theta=\pi$. Thus the $p g f$ gives a more realistic impression of the actual probability distribution. See [6] for a graphic example.

Each $\mathrm{C}_{\mathrm{m}}(\mu, \delta)$ distribution is isotropic about its mean axis, $\pm \mu$. Watson [7] has developed an array of statistical methods for analyzing data samples from isotropic directional distributions of arbitrary dimensions that is suitable for $\mathrm{C}_{\mathrm{m}}(\mu, \delta)$ distributions.

Cauchy $m$-sphere distributions possess an interesting duality feature, namely:

$\theta, S, x \propto \mathrm{C}_{\mathrm{m}}(\mu, \delta) \Leftrightarrow \theta, S, x \propto \mathrm{C}_{\mathrm{m}}\left(-\mu, \delta^{-1}\right)$

To see this, specify the argument of $A$ as a function of $\delta$ or $\delta^{-1}$ by $A(\delta)$ or $A\left(\delta^{-1}\right)$ and likewise for $B$, in $(9)$. Then it is readily verified that $\mathrm{A}(\delta)-B(\delta) x \mu^{\mathrm{T}}=A\left(\delta^{-1}\right)-B\left(\delta^{-1}\right) x(-\mu)^{\mathrm{T}}$, and (12) follows.

Another interesting feature is that $A+B=\delta$ and $A-B=$ $\delta^{-1}$ which, in conjunction with (11), can be used to find the ratio of the maximum pgf values to their corresponding minimum values for any Cauchy $\mathrm{C}_{\mathrm{m}}(\mu, \delta)$ distribution (the ratio is either $\delta^{2}$ or $\delta^{-2}$ according as $\delta>1$ or $\left.\delta<1\right)$. This ratio is the same $\left(\delta^{2}\right.$ or $\left.\delta^{-2}\right)$ for all dimensions $m$ and any mean direction $\mu$ on $S_{\mathrm{d}}$. The corresponding ratio for the family of Fisher-von Mises distributions is $\exp (2 \kappa)$ for all dimensions and all mean directions (see [8] for relevant properties of the Fisher-von Mises distributions).

\section{STEREOGRAPHIC PROJECTION}

\subsection{Rotating m-Spheres into Polar Positions}

At times it is necessary to rotate $\mu$ to a polar mean position $\pm \mathrm{n}$, which is the fixed position for an $m$-sphere $S$ with the added proviso that $\mu= \pm n$, so that

$S \sim \mathrm{C}_{\mathrm{m}}( \pm \mathrm{n}, \delta)$, say.

The transpose $\mathrm{u}^{\mathrm{T}}$ of any input point $\mathrm{u}$ (except $\mathrm{u}= \pm \mathrm{n}$ ) on $S$ is rotated to the transpose $\mathrm{v}^{\mathrm{T}}$ of the output point $\mathrm{v}$ of any other given point v on $S$ by the $n \times n$ orthogonal matrix $Q$ given by

$Q \mathrm{u}^{\mathrm{T}}=\left\{(\mathrm{u}+\mathrm{v})^{\mathrm{T}}\left(1+\mathrm{uv}^{\mathrm{T}}\right)^{-1}(\mathrm{u}+\mathrm{v})-I_{\mathrm{n}}\right\} \mathrm{u}^{\mathrm{T}}=\mathrm{v}^{\mathrm{T}}$

where $I_{\mathrm{n}}$ is the $n \times n$ identity matrix. The orthogonal matrix $Q$ is symmetric and thus equal to its own inverse. It may, among other things, be used to rotate $\mu$ into or out of polar mean positions.

The poles, \pm n, of any $m$-sphere $S$ are considered as fixed and immutable, with $\mathrm{n}$ at the top of the polar axis, $\mathrm{s}$ at the bottom, and the polar axis orthogonal to the equatorial $m$-space $\mathrm{E}_{\mathrm{q}}$ spanned by $x_{2} \ldots x_{\mathrm{n}}$. It is necessary, before doing any projections, to ensure this positioning of the polar axis and the equatorial $m$-space $\mathrm{E}_{\mathrm{q}}$. It may also be necessary to rotate $\mu$ to $\mathrm{n}$.

\subsection{Projective Mappings, Images, and Spaces}

The equator of $S$ is the unit (m-1)-sphere orthogonal to the polar axis of $S$. The equatorial $m$-space $\mathrm{E}_{\mathrm{q}}$ of $S$ is the set 
of points $y$ with the form $\left(\begin{array}{lll}0 & x_{2} \ldots x_{\mathrm{n}}\end{array}\right)$ that are orthogonal to the polar $\mathrm{x}_{1}$-axis, the exact same set of points as the m-space $L$ (or $L_{\mathrm{m}}$ ) spanned by the centerlines of the polar circles of $S$. All these linear point sets are extended sets, with the single improper point $\infty$ appended to each and every line in them. All parallel lines of $L$ are extended lines, and they all meet at infinity; indeed, all the lines of $L$ are extended and meet at infinity. For more details about the improper point $\infty$ see [9-12].

In this section stereographic projection is extended beyond the usual 2- and 3-dimensional mappings between circles and lines, and spheres and planes. We use real $n$-vectors, $x$ and $y$, on $m$-spheres $S$ and on their centerline m-spaces $L$. We will construct a projective mapping $\mathrm{P}_{\mathrm{LS}}$ from $S$ to $L: L=$ $\mathrm{P}_{\mathrm{LS}} S$, which projects the unit vectors $x$ on $S$ to the points $y$ of $L$ in a one-one and onto fashion: $y=\mathrm{P}_{\mathrm{LS}} x$. Since this projective mapping must be one-one and onto, it implies the consequent result that $L=\mathrm{P}_{\mathrm{LS}} S$, with inverse mapping given by $S=\mathrm{P}_{\mathrm{SL}} L$, say. In view of the above we may say that $L$ and $S$ (and $x$ and $y$ ) are projective images of one another, and write:

$\left[L \leftrightarrow S \Leftrightarrow L=\mathrm{P}_{\mathrm{LS}} S\right] \Leftrightarrow\left(y=\mathrm{P}_{\mathrm{LS}} x\right.$ and $\left.x=\mathrm{P}_{\mathrm{SL}} y\right)$

in a one-one and onto fashion, for all $x$ in $S$ and $y$ in $L$. A more precise definition of "image" is this:

For the projective mapping $\mathrm{y}=\mathrm{P}_{\mathrm{LS}} \mathrm{x}$ and its implied result $\mathrm{L}=\mathrm{P}_{\mathrm{LS}} \mathrm{S}$, the points $\mathrm{x}$ and $\mathrm{y}$ are called projective images (or images, for short) of one another, and we write: $\mathrm{y} \leftrightarrow \mathrm{x}$, when $x$ and $y$ are collinear with the north pole $n$. If $(n, x, y)$ are collinear then so are $(\mathrm{n}, \mathrm{y}, \mathrm{x})$, so we may also write $\mathrm{x} \leftrightarrow \mathrm{y}$.

The fixed north pole $n$ on $S$ is called the projection point of the mapping, And the image relation is an equivalence relation, being reflexive, symmetric and transitive.

All points on $S$ or $L$ or $\mathrm{E}_{\mathrm{n}}$ are row n-vectors. The $\mathrm{m}$ sphere $S$ has definition $\left.S=\left\{x: x=\left(x_{1} \ldots x_{\mathrm{n}}\right),|x|=1\right)\right\}$ (ext), and $S=\left\{a: a=\left(1, a_{1} \ldots a_{\mathrm{m}}\right)\right\}$ (int, because it is expressed in terms of its internal spherical coordinates $a$ ).

$L$ has extrinsic definition: $L=\left\{\mathrm{y}: \mathrm{y}=\left(0 \mathrm{x}_{2} \ldots \mathrm{x}_{\mathrm{n}}\right)\right\}$ (ext) and intrinsic definition $L=\left\{\mathrm{y}: \mathrm{y}=\left(0 \mathrm{y}_{2} \ldots \mathrm{y}_{\mathrm{n}}\right)\right\}$ (int). The extended linear $\mathrm{n}$-space is defined by $\mathrm{E}_{\mathrm{n}}=\left\{x: x=\left(x_{1} \ldots x_{\mathrm{n}}\right),-\infty\right.$ $\left.<x_{\mathrm{j}} \leq \infty\right\}$ (int).

We proceed to derive the projective mapping $\mathrm{P}_{\mathrm{LS}}$ which maps every point $x$ on the finite curved $m$-sphere $S$, in a oneone and onto manner, to a point $y$ on the infinite linear $m$ space of $L$.

\subsection{A Projective Role for Cauchy Distributions}

Any unit circle $C$ can be stereographically projected to its extended centerline $\mathrm{L}$ by the one-one and onto mapping $y$ $=\cot a / 2$, where $y \in \mathrm{L}, x=(\sin a, \cos a) \in C$, and $a$ is a suitably defined angular deviation of $x$ from $\mathrm{n}$. The value of $y \in(-\infty, \infty]$ as $x$ moves around the circle. This example is extended to bring linear and circular Cauchy distributions into the picture. Recall that a point $y$ varying over a line $\mathrm{L}$, and/or L itself, are said to be linear Cauchy distributed, with median $v$ and scale parameter the rescaler $\delta$, over the centerline L, and we write: $y$ or $\mathrm{L} \sim \mathrm{L}_{1}(v, \delta)$, when $y$ has probability density function

$\operatorname{pdf}(y)=(\delta \pi)^{-1}\left[1+\{(y-v) \delta\}^{2}\right]^{-1}$ where $\delta>0$

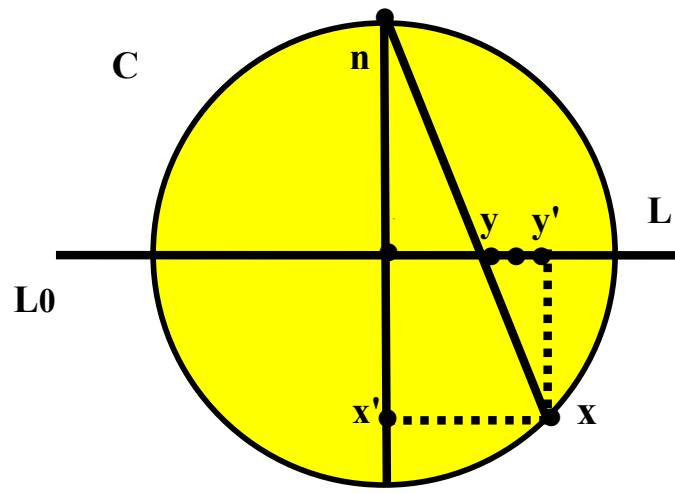

$\mathbf{S}$

Fig. (2). Planar Cross-Section of the $m$-sphere $S$ and centerline m-space $L$ for the Projective Mapping $y=\mathrm{P}_{\mathrm{LS}} x$. The cross-section of the $m$-sphere $S$ is the unit circle $C$, and the cross section of the $m$-space $L$ is the horizontal centerline L. The three points $\mathrm{n}, x, y$ in Fig. 2 are collinear, so $x=\left(x_{1} \ldots x_{\mathrm{n}}\right)$ (ext) and $y=\left(0 y_{2} \ldots y_{\mathrm{n}}\right)$ (int) are images, and we write: $x \leftrightarrow y$.

We say $y$ or $\mathrm{L}$ is standard Cauchy and write: $y$ or $\mathrm{L} \sim$ $\mathrm{C}_{1}(0,1)$, when the median $v$ is zero and the rescaler is unity. It is well-known that

$\mathrm{L} \leftrightarrow \mathrm{C} \Rightarrow\left\{\mathrm{L} \sim \mathrm{L}_{1}(0,1) \Leftrightarrow \mathrm{C} \sim \mathrm{C}_{1}(0,1)\right]$

This states that if a projective mapping $\mathrm{P}_{\mathrm{LC}}$ exists between a unit circle $\mathrm{C}$ and its extended centerline $\mathrm{L}$, then a uniform distribution on $\mathrm{C}$ induces a standard Cauchy distribution on L, and conversely. Downs [1,\$3.1] used the change of variables theorem to generalize (16) to

$\mathrm{L} \leftrightarrow \mathrm{C} \Rightarrow\left[\mathrm{L} \sim \mathrm{L}_{1}(0, \delta) \Leftrightarrow \mathrm{C} \sim \mathrm{C}_{1}\left(\mu_{\delta}, \delta\right)\right]$

where $\mu_{\delta}=(\mathrm{s}, 0, \mathrm{n})$ according as $\delta$ is $(<1,=1,>1)$.

This is an interesting result. It states that, if $\mathrm{L}$ and $\mathrm{C}$ are images of one another as in (16), then rescaling $\mathrm{L}$ by $\delta$ induces a Cauchy $\mu_{\delta}, \delta$ distribution on $\mathrm{C}$, and vice versa, thus creating a non-uniform Cauchy distribution from scratch with (17) via an unadulterated line and circle in (16). We can even use (17) to create an arbitrary circular Cauchy $\mu, \delta$ from scratch by rotating $\mu_{\delta}$ in (17) to $\mu$, via (13).

To extend these results, define the $m$-space $L$ to be Cauchy $v, \delta$ over an extended $m$-space $L$, and write:

$L \sim \mathrm{L}_{\mathrm{m}}(v, \delta)$ when every line $\mathrm{L}$ in $L$ is $\mathrm{L}_{1}(v, \delta)$.

We claim now- and prove later-a remarkable extension of (17):

$L \leftrightarrow S \Rightarrow\left[L \sim \mathrm{L}_{\mathrm{m}}(0, \delta) \Leftrightarrow S \sim \mathrm{C}_{\mathrm{m}}\left(\mu_{\delta}, \delta\right)\right]$

This result enables us to convert an infinite linear mspace $L$ to a finite curved m-space $S$, and conversely, for any positive integer $m$. However, we must first prove the antecedent that $S$ and $L$ are projective images: $S \leftrightarrow L$. We proceed to do just that.

We seek a projective mapping $\mathrm{P}_{\mathrm{LS}}$ which sends every point $x$ of an m-sphere $S$ to a point $y$ in an m-space $L$ in a one-one and onto manner, so (14) is satisfied and $L$ and $S$ are indeed projective images of one another. First, simplify the complicated m-spaces of $L$ and $S$ to 1 -spaces $\mathrm{L}$ and $C$ using 
the fact that all the action stems from the three special points n, s, $x$ on the surfaces of $S$ and the unit circle $C$ (Fig. 2).

The three special points are the poles $\mathrm{n}$ and s of $S$ and $C$, and a randomly selected non-polar point $x$ on $S$. These special points fix the plane containing $C$, and serve to define $C$ as the unique polar circle cross-section of $S$ which contains $x$. The centerline $\mathrm{L}$ of $C$ is the corresponding cross-section of $L$. The point $x$ on $S$ has unit length and so must be on $C$. To construct $C$ draw a vertical polar axis for $S$ and for $C$ two units in length. Label the top, middle and bottom points as n, 0 and s (north pole, origin, and south pole), thus determining $C$ (Fig. 2). The m-sphere $S$ has been reduced to the crosssection of $S$ containing the four points $\mathrm{n}, \mathrm{s}, x$ and $y$. The equatorial $\mathrm{m}$-space $\mathrm{E}_{\mathrm{q}}=L_{\mathrm{m}}$ has been reduced to the centerline $\mathrm{L}$ of $C$.

The perpendicular projections of $x$ onto the polar axis $\pm \mathrm{n}$ and the line $\mathrm{L}$ yield the auxiliary points $x^{\prime}$ and $y^{\prime}$ respectively. From the rectangle with vertices $0, x^{\prime}, x, y^{\prime}$ we get $x=x^{\prime}+y^{\prime}$, or $\left(x_{1} \ldots x_{\mathrm{n}}\right)(\mathrm{ext})=\left(x_{1} 0 \ldots 0\right)(\mathrm{ext})+\left(0 x_{2} \ldots x_{\mathrm{n}}\right)(\mathrm{ext})$. Note that $x_{1}$ $<0$ since $x$ is below $\mathrm{L}$, and that $y-y^{\prime} \| x-x^{\prime}$. As $x$ varies over $S$, the circle $C$ and its image line $\mathrm{L}$ change correspondingly. The points $y$ of each centerline are mapped one-one and onto the points $x$ of its corresponding polar circle, thanks to the extension to $\infty$ of the lines of $\mathrm{L}$.

If $x$ lies above $\mathrm{L}$ then so does $x^{\prime}$, and $x$ is then between $\mathrm{n}$ and $y$, with $y$ still on L but outside of C. But the fundamental structure of the point configuration of Fig. (2) remains intact. Analogous configurations occur when $x$ is to the left of the polar axis.

Some features of the mapping $\mathrm{P}_{\mathrm{LS}}$ become apparent. The north pole $\mathrm{n}$ is mapped to the improper point $\infty$ of $\mathrm{L}$, and the south pole s to the origin 0 . The equator of $S$ is mapped to itself. Since $\mathrm{E}_{\mathrm{q}}$ is orthogonal to the polar axis of $S_{\mathrm{m}}$ then every line $\mathrm{L}$ through 0 in $\mathrm{E}_{\mathrm{qm}}$ must be orthogonal to the polar axis.

The mapping $y=\mathrm{P}_{\mathrm{LS}} x$ sends the point $x$ on the finite mdimensional curved surface of $S$ to the point $y$ on the infinite m-dimensional linear space $L$, and conversely for $\mathrm{P}_{\mathrm{SL}}$. The point $x=\left(x_{1} \ldots x_{\mathrm{n}}\right)$ on $S_{\mathrm{m}}$ is restricted by $x x^{\mathrm{T}}=1$, but its image point $y=\left(0 y_{2} \ldots y_{\mathrm{n}}\right)$ on $\mathrm{E}_{\mathrm{q}}$ is unrestricted. The first coordinate $y_{1}$ of $y$ is always 0 for all $x$.

We seek an extrinsic form for $y$ as a function of $x$. To this end we derive the mappings $\mathrm{P}_{\mathrm{SL}}$ and $\mathrm{P}_{\mathrm{LS}}$ to prove their existence. For $\mathrm{P}_{\mathrm{LS}}$ this is done by expressing each element of $y$ as a function of the elements of $x$, and conversely for $P_{\mathrm{SL}}$. We show, with heavy reliance on Fig. (2), that:

$y=\left(0 y_{1} \ldots y_{\mathrm{n}}\right)($ int $)=\mathrm{P}_{\mathrm{LS}} x=y^{\prime} /\left(1-x_{1}\right)(e x t)$

where $y^{\prime}=\left(0 x_{2} \ldots x_{\mathrm{n}}\right)$. Note the equation $x_{1}=0$ is an extrinsic definition of the equatorial $m$-space $L=\mathrm{E}_{\mathrm{q}}$.

To show (19) observe that the right triangles with hypotenuses through the points $(\mathrm{n}, y)$ and $(\mathrm{n}, x)$ are similar, so $|y| /|\mathrm{n}|=\left|x-x^{\prime}\right| /\left(|\mathrm{n}|+\left|x^{\prime}\right|\right)$, which is the same as $|y| / 1=$ $\left|y^{\prime}\right| /\left(1-x_{1}\right)$ (because $x_{1}$ is negative), and this is the same as $|y| /\left|y^{\prime}\right|=1 /\left(1-x_{1}\right)$. The points $x, y$ and $y^{\prime}$ are always on the same side of the polar axis, implying that $y$ and $y^{\prime}$ are always parallel with the same sense (both east of 0 in Fig. 2), implying equality of the unit $n$-vectors: $y /|y|$ and $y^{\prime} /\left|y^{\prime}\right|$. Since $|y| /\left|y^{\prime}\right|=1 /\left(1-x_{1}\right)$, we infer that $y=y^{\prime} \times|y| /\left|y^{\prime}\right|=y^{\prime} /\left(1-x_{1}\right)$, which proves (19). Observe that (19) is valid regardless of the quadrant in which $x$ lies on $C$.

The final step is to find, for each image pair $(x, y)$, an extrinsic representation of $x$ in terms of intrinsic elements of $y$ for the inverse mapping $P_{\mathrm{SL}}$. Let the point $y=\left(0 y_{2} \ldots y_{\mathrm{n}}\right) \in L_{\mathrm{m}}$ be assigned. We must find

$x($ ext $)=\mathrm{P}_{\mathrm{SL}} y$, where $y=\left(0 y_{2} \ldots y_{\mathrm{n}}\right)($ int $)$.

Assume the reasonable conjecture that the form for $\mathrm{x}=$ $\mathrm{P}_{\mathrm{SL}} \mathrm{y}$ when $n=3$ is the same as that when $n$ is arbitrary. If $n$ is 3 the known form (see [9]) is:

$x=P_{\mathrm{SL}} y=\left(\mathrm{q}^{2}-12 y_{2} 2 y_{3}\right) /\left(\mathrm{q}^{2}+1\right)$, and $\mathrm{q}^{2}=y_{2}{ }^{2}+\mathrm{y}_{3}{ }^{2}$.

For arbitrary $n$, the form is:

$x=P_{\mathrm{SL}} y=\left(\mathrm{q}^{2}-12 y_{2} \ldots 2 y_{\mathrm{n}}\right) /\left(\mathrm{q}^{2}+1\right), \mathrm{q}^{2}=\Sigma y_{\mathrm{j}}^{2}$

where the sum is for $\mathrm{j}=2$-n. Initially test the conjecture by checking that $|x|^{2}=1$. By (20)

$\left.x x^{\mathrm{T}}=\left\{\left(\mathrm{q}^{2}-1\right)^{2}+4 \mathrm{y}_{2}{ }^{2}+\ldots+4 \mathrm{y}_{\mathrm{n}}{ }^{2}\right)\right\} /\left(\mathrm{q}^{2}+1\right)^{2}$.

The numerator of this is $\left(q^{2}-1\right)^{2}+4 q^{2}=q^{4}-2 q^{2}+1+4 q^{2}$ which is $\left(\mathrm{q}^{2}+1\right)^{2}$, the denominator. The quick initial test is met. The decisive test is: does $P_{\mathrm{SL}} P_{\mathrm{LS}} x=x$ ? Since $x^{\prime}=(0$ $\left.x_{2} \ldots x_{\mathrm{n}}\right)$, then

$$
y=\mathrm{P}_{\mathrm{yx}} x=y^{\prime} /\left(1-x_{1}\right)(e x t)=\left(0 x_{2} \ldots x_{\mathrm{n}}\right) /\left(1-x_{1}\right)(e x t) \text { by }
$$
(19). By the conjecture (20) we have:

$$
\begin{aligned}
x= & \mathrm{P}_{\mathrm{SL}} y=\left(x_{1} \ldots x_{\mathrm{n}}\right)=\left(\mathrm{q}^{2}-12 \mathrm{y}_{2} \ldots 2 \mathrm{y}_{\mathrm{n}}\right)\left(\mathrm{q}^{2}+1\right) \\
& \text { with } \mathrm{q}^{2}=\mathrm{y}_{2}{ }^{2}+\ldots+\mathrm{y}_{\mathrm{n}}{ }^{2}, \text { implying } \\
x_{1}= & \left(\mathrm{q}^{2}-1\right)\left(\mathrm{q}^{2}+1\right), \text { and } y_{\mathrm{j}}=x_{\mathrm{j}}\left(1-x_{1}\right) \text { for } \mathrm{j}=1-\mathrm{n} .
\end{aligned}
$$

Solve the equation $x_{1}=\left(\mathrm{q}^{2}-1\right)\left(\mathrm{q}^{2}+1\right)$ for $\mathrm{q}^{2}$ and get

$\mathrm{q}^{2}=\left(1+x_{1}\right) /\left(1-x_{1}\right)$, so $\mathrm{q}^{2}+1=2 /\left(1-x_{1}\right)$, and, by $(21)$

$2 y_{\mathrm{j}} /\left(\mathrm{q}^{2}+1\right)=\left\{2 x_{\mathrm{j}} /\left(1-x_{1}\right)\right\} \times\left\{\left(1-x_{1}\right) / 2\right\}=x_{\mathrm{j}}$,

for $\mathrm{j}=2-n$, confirming that $x=P_{\mathrm{SL}} P_{\mathrm{LS}} x$ and completing the proof that the $m$-spaces $S$ and $L$ are projective images of one another. This satisfies the antecedent condition in (18) that $S \leftrightarrow L$, and sets the stage for proving (18).

\subsection{Proof of $L \leftrightarrow S \Rightarrow\left[L \sim \mathrm{L}_{\mathrm{m}}(0, \delta) \Leftrightarrow S \sim \mathrm{C}_{\mathrm{m}}\left(\mu_{\delta}, \delta\right)\right]$}

We must prove $L \sim \mathrm{L}_{\mathrm{m}}(0, \delta) \Leftrightarrow S \sim \mathrm{C}_{\mathrm{m}}\left(\mu_{\delta}, \delta\right)$, given that $L \leftrightarrow S$. First assume that $L \leftrightarrow S$ and $S \sim \mathrm{C}_{\mathrm{m}}\left(\mu_{\delta}, \delta\right)$. We must prove that $L \sim \mathrm{L}_{\mathrm{m}}(0, \delta)$ :

Proof: The set of centerlines $\mathrm{L}$ in $L$ span $L$; the set of polar circles $C$ in $S$ span S; every $\mathrm{L}$ in $L$ has a unique polar circle $C$ in $S$ : the lines and circles are paired off as $(\mathrm{L}, C)$; and the continuum of $(\mathrm{L}, C)$ pairs span the pair of m-spaces $(L, S)$.

Since $S \sim \mathrm{C}_{\mathrm{m}}\left(\mu_{\delta}, \delta\right)$ for all pairs $(\mathrm{L}, C)$ the $C$ s must be identically distributed as $\mathrm{C}_{1}\left(\mu_{\delta}, \delta\right)$ with identical mean $(\mathrm{s}, 0$, or $\mathrm{n})$ by (11). Then the corresponding Ls for the $C$ s are identically distributed as $\mathrm{L}_{1}(0, \delta)$ by $(17)$, and $L$ is $\mathrm{L}_{\mathrm{m}}(0, \delta)$ by definition.

Assume $L \sim \mathrm{L}_{\mathrm{m}}(0, \delta)$. We now show $S \sim \mathrm{C}_{\mathrm{m}}\left(\mu_{\delta}, \delta\right)$ :

Proof: The centerlines $\mathrm{L}$ of $L$ are identically distributed as $\mathrm{L}_{1}(0, \delta)$ by definition of $\mathrm{L}_{\mathrm{m}}(0, \delta)$. Then, by (17), $C \sim \mathrm{C}_{1}\left(\mu_{\delta}\right.$, $\delta$ ) for every polar circle $C$ on $S$. This implies the m-sphere $S$ 
has profile generating function $\operatorname{pgf}(S) \propto\left(A-B x \mu_{\delta}^{\mathrm{T}}\right)$ where $x$ $\in S$, implying by (11) and the discussion following it that $S \sim$ $\mathrm{C}_{\mathrm{m}}\left(\mu_{\delta}, \delta\right)$. This completes the proof of (18).

There are two fixed points for $S_{\mathrm{m}}$ and two for $L_{\mathrm{m}}$ in every stereo-projective mapping between them: the fixed points for $S_{\mathrm{m}}$ are the poles $\mathrm{n}$ and $\mathrm{s}$ on $\mathrm{S}_{\mathrm{m}}$, and their respective images are the fixed points $\infty$ and 0 of $L_{\mathrm{m}}$, since these fixed points are inviolate and cannot be changed.

The crucial role of $\infty$ in this process legitimizes and solidifies the conclusions that the projective mapping from the curved surface of $S_{\mathrm{m}}$ to the linear surface of $L_{\mathrm{m}}$ is indeed a one-one and onto mapping.

\section{TRANSFORMATIONS}

Changing $\mu$ to $\mu^{\prime}$, say, is done by rotating $\mu^{\mathrm{T}}$ to $\mu^{\prime T}$. Changes in scale from $\delta$ to $\delta^{\prime}$ are done by rescaling the coordinate axes from $\delta$ to 1 , then from 1 to $\delta^{\prime}$, or by simply rescaling the $x_{2} \ldots x_{\mathrm{n}}$ coordinate axes by $\left(\delta^{\prime} / \delta\right)$. A variety of transformations can be achieved by deft manipulation of (16)-(18).

Circular Cauchy distributions are amenable to sequences of simultaneous transformations of location and scale parameters because a special class of complex-valued Möbius transformations form a subgroup under composition of transformations. This subgroup is composed of Cauchy circle mappings from one planar unit circle to itself or to another unit circle, with the resulting circular Cauchy distributions having transformed parameters. For further details using complex variables see $[1 ; \S 2,3.4]$.

The spherical Cauchy distributions are closed under all Möbius transformations, with results analogous to those for circular Cauchy distributions. For further details (in a complex plane setting) see $[1 ; \S 2,4,4.8]$.

The evidence is mounting that a family of spherical distributions is closed under Möibus transformations if and only if that family is Cauchy-based.

Analogous evidence has accumulated for circular Cauchy distributions when the Möbius mappings are confined to the subgroup of circle mappings. See [1, § $2 \& 4]$ for more details.

\section{DISCUSSION}

The techniques of pattern recognition and image analysis are heavily dependent on sophisticated statistical methods, large databases and extensive computations. Bahlmann [13] has pointed out situations where directional and linear data concurrently appear in these fields but where there is no integrated body of statistical techniques that provide an integrated solution to this problem of disparate data domains. The family of Fisher-von Mises distributions has been extended from domains of observations of vectors on $\mathrm{m}$ - spheres, to domains where the observations are rigid configurations of $\mathrm{p}$ distinguishable directions in $\mathrm{n}$-space, $p \leq n$ [14]. He applied this successfully to analyses of the orientations of spatial QRS loops observed from clinical vectorcardiograms, each with 2 vectors in 3 -space $(p=2, n=3)$. These methods show promise for application in such areas as pattern recognition and image analysis that possess endless varieties of shapes and configurations.

The Cauchy families abound in hyperspherical and linear domains, and Cauchy objects in either of these domains can be simply transformed to Cauchy objects in the other domain, thus eliminating the problem of disparate data domains. All Cauchy-based objects in either domain are amenable to changes in location and scalar parameters.

\section{CONFLICT OF INTEREST}

The authors confirm that this article content has no conflicts of interest.

\section{ACKNOWLEDGEMENT}

Declared none.

\section{REFERENCES}

[1] T. D. Downs, "Cauchy families of directional distributions closed under location and scale transformations", Open Stat. Probab., vol. 1, pp.76-92, 2009. [Online] Available: benthamscienceopen/tospj [Accessed 23 ${ }^{\text {rd }}$ Dec 2011].

[2] Wikipedia, [Online] Mathematics, n-sphere, Hyperspherical coordinates. [Accessed 23 ${ }^{\text {rd }}$ Dec 2011].

[3] C.H. Edwards, Jr. Advanced Calculus of Several Variables, New York: Dover 1994, pp. 261-263.

[4] I. S. Gradshteyn, and I. M. Ryzhik, Table of Integrals, Series, and Products. Academic Press: New York, 1980. [Corrected and Enlarged Edition prepared by Alan Jeffrey, pp. 378-379].

[5] T. D. Downs, and A.L. Gould, "Some relationships between the normal and von Mises distributions", Biometrika, vol. 54, no. 3 and 4, pp. 684-686, 1967.

[6] K.V. Mardia, Statistics of Directional Data. Academic Press: London 1972, p. 229.

[7] G.S. Watson, Statistics on Spheres. John Wiley \& Sons: New York, 1983, [Chapters 4, 5].

[8] T. D. Downs, "Some relationships among the von Mises distributions of different dimensions", Biometrika, vol. 53, pp. 269-272. 1966.

[9] T. Needham, Visual Complex Analysis, Oxford University Press: New York 1998, pp. 139-153.

[10] H. Schwerdtfeger, Geometry of Complex Numbers. Dover: New York 1979, p. 120.

[11] P. McCullagh, "Möbius transformation and Cauchy parameter estimation", Ann. Stat., vol. 24, no. 2, pp. 787-818, 1996. [Online] Available at: http://projecteuclid.org/aos [Accessed: $2^{\text {nd }}$ Dec, 2011].

[12] Wikipedia, Stereographic Projection. [Accessed $23^{\text {rd }}$ June. 2012].

[13] C. Bahlmann, "Directional features in online handwriting recognition”, Pattern Recognit., vol. 39, pp. 115-125, 2006. [Online] Available: sciencedirect.com [Accessed: $20^{\text {th }}$ June, 2012].

[14] T. D. Downs, "Orientation statistics", Biometrika, vol. 59, pp. 665$676,1972]$

Received: May 12, 2012

Revised: June 25, 2012

Accepted: June 28, 2012

(C) Downs and Downs; Licensee Bentham Open.

This is an open access article licensed under the terms of the Creative Commons Attribution Non-Commercial License (http://creativecommons.org/licenses/by-nc/3.0/) which permits unrestricted, non-commercial use, distribution and reproduction in any medium, provided the work is properly cited. 